\title{
Motility and percentage of Y- and YY-bearing spermatozoa in human semen samples after passage through bovine serum albumin
}

\author{
G. David, C. Jeulin, A. Boyce* and D. Schwartz* \\ Laboratoire d'Histologie-Embryologie-Cytogénétique, Centre Hospitalier de Bicêtre, \\ 78 rue du Général Leclerc, 94270 Kremlin-Bicêtre and \\ *Unité de Recherches Statistiques de l'I.N.S.E.R.M., \\ 16 bis Avenue Paul-Vaillant-Couturier, 94800 Villejuif, France
}

The possibility of increasing the percentage motility and the number of Y-bearing spermatozoa by passage through graduated layers of bovine serum albumin (BSA) was first reported by Ericsson, Langevin \& Nishino (1973), but their conclusions have become the subject of controversy (Ross, Robinson \& Evans, 1975; Evans, Douglas \& Renton, 1975). We therefore undertook a series of experiments, some involving the techniques described by Ericsson et al. (1973), in an attempt to clarify this issue.

The semen used throughout was collected at the laboratory either from donors for artificial insemination or from patients who had been referred because of childlessness but whose semen was judged to be normal ( $>50 \times 10^{6}$ spermatozoa $/ \mathrm{ml}$ and $60 \%$ motile spermatozoa). For each sample of fresh semen and those from the isolation layers the percentage motility of spermatozoa was estimated by a conventional method, and the $\mathrm{Y}$-bearing spermatozoa were identified by the quinacrine staining technique (Barlow \& Vosa, 1970; Sumner, Robinson \& Evans, 1971). For the latter, a 1\% (w/v) quinacrine dihydrochloride (Sigma) solution was made up in a buffer solution of $0.1 \mathrm{M}$-sodium citrate and $0.2 \mathrm{M}$-disodium phosphate, $\mathrm{pH} 5.5$. The staining time was $15 \mathrm{~min}$. The $\mathrm{Y}$ count was performed separately by two observers and it included those spermatozoa which had 2 F-bodies; none was found to have more than two. The slides presented to the observers were not selected beforehand and no slide was rejected by the observers.

In the first experiment, 9 subjects were studied ( 5 patients and 4 donors, 1 donor being used twice) and the one-step technique was used with BSA concentrations of 10,15 and $25 \%$. The Y counts were performed on about 300 spermatozoa from each fraction. Overall, a clear increase was observed, both in the percentage motility $(92.7 \pm 2.9 \%$ (S.E.M.) versus $56.5 \pm 3.1 \%$ ) and in the percentage of Y-bearing spermatozoa $(55 \cdot 3 \pm 1 \cdot 4 \%$ versus $41 \cdot 5 \pm 1 \cdot 7 \%$, both these increases being highly significant $\left(t=9 ; 9\right.$ d.f.; $\left.P<10^{-5}\right)$. However, when the $Y$ count was analysed, an observer effect and a time trend were noted. During the 5 months of the experiment the $Y$ count for the fresh semen samples had increased progessively from approximately $35 \%$ to $45 \%$, whilst the difference between observers diminished steadily. It is clear, therefore, that the $\mathrm{Y}$ count is a somewhat subjective operation, as discussed by Beatty (1974a), which may explain certain low counts reported in the literature. Although, for the most part, this subjectivity was eliminated as the counts were performed on the same day by the same observer for the fresh and the isolated spermatozoa, there still remained some doubt about the conclusions which could be drawn because no blind precautions had been taken.

A second one-step experiment was therefore undertaken, a single subject being used and several slides being prepared for each sample of spermatozoa to be examined. On each of 4 different days a set of slides from the different fractions was stained and examined in randomized order by both observers, each count being performed blind on 200 spermatozoa. An analysis of variance was performed on the data which are summarized in Table 1 , the following factors being taken into account: observers ( 1 d.f.), day of count ( 3 d.f.), fractions ( 5 d.f.). There was no observer effect and no difference between repeat counts, but a highly significant difference in the percentage of $Y$ spermatozoa in the different fractions was obtained $\left(\mathrm{F}_{5,38}=29 \cdot 9 ; P<10^{-9}\right)$. 
Table 1. Results of an experiment with human semen (one subject) separated into fractions* by the one-step technique of Ericsson et al. (1973)

\begin{tabular}{lcccccc}
\hline & $\begin{array}{c}\text { Fresh } \\
\text { semen }\end{array}$ & $\begin{array}{c}\text { Washed } \\
\text { sperm. }\end{array}$ & Top layer & $10 \%$ BSA & $15 \%$ BSA & $25 \%$ BSA \\
\hline$\%$ Y count & $41 \cdot 0$ & $42 \cdot 5$ & $42 \cdot 2$ & $40 \cdot 2$ & $53 \cdot 1$ & $54 \cdot 3$ \\
$\%$ Motility & 65 & 50 & $35-40$ & 60 & $90-95$ & 95 \\
\hline
\end{tabular}

* The Y counts on 200 spermatozoa were repeated blind on 4 different days by 2 observers (i.e. 1600 spermatozoa from each fraction).

Table 2. Results of an experiment with human semen (one subject) separated into fractions* by the three-step technique of Ericsson et al. (1973)

\begin{tabular}{lcccc}
\hline & Washed sperm. & $10 \%$ BSA & $15 \%$ BSA & $20 \%$ BSA \\
\hline$\%$ Y count & 48.4 & $53 \cdot 8$ & $58 \cdot 8$ & $56 \cdot 3$ \\
$\%$ Motility & $60-65$ & $90-95$ & 95 & 95 \\
\hline
\end{tabular}

* See Table 1 for details of counts.

With the same experimental conditions as in Exp. 2, a third experiment was performed, but the three-step technique of Ericsson et al. (1973) was used. Again there was no observer effect and no difference between repeat counts (Table 2$)$, but there was a difference between the fractions $\left(F_{3,24}=\right.$ $6.8 ; P<0.01$ ).

In these three experiments, the increase in percentage motility is clear, but it was realized that the counts showing the enrichment of fractions with $\mathrm{Y}$-bearing spermatozoa were not truly blind because it was possible to distinguish the various samples by the difference in sperm concentration. Two more experiments were therefore undertaken to measure the extent of any possible bias.

The first of these two experiments consisted of a simulation in which series of dilutions were presented to the observers as being samples drawn from the isolation layers of a separation experiment. The counts were performed on 400 spermatozoa from each fraction and those obtained for all the dilutions were similar to that obtained for the undiluted semen:

$\begin{array}{lccccc}\text { Sperm count }\left(\times 10^{6} / \mathrm{ml}\right) & \begin{array}{c}155 \\ \text { (undiluted) }\end{array} & 150 & 75 & 37 & 18 \\ \% \text { Y count }( \pm 2 \text { S.E. }) & 45 \cdot 5 \pm 5 \cdot 0 & 50 \cdot 0 \pm 5 \cdot 0 & 48 \cdot 5 \pm 5 \cdot 0 & 47 \cdot 0 \pm 5 \cdot 0 & 47 \cdot 0 \pm 5 \cdot 0\end{array}$

In the second of these experiments the observers were told that the slides consisted of series of dilutions, whereas in fact they were a mixture of dilutions and samples from the isolation layers of a one-step separation experiment on a single column of $20 \%$ BSA. This column was divided into three equal layers which were pipetted off separately. The results given below show clearly that there was no increase in $\mathrm{Y}$ count with dilution, whereas there was a clear increase after passage through BSA. The counts were performed on 1000 spermatozoa except in the middle BSA layer when 500 only were counted.

\begin{tabular}{|c|c|c|c|c|c|}
\hline & \multirow{2}{*}{ Fresh semen } & \multicolumn{2}{|c|}{ Dilution } & \multicolumn{2}{|c|}{ BSA } \\
\hline & & $20 \times 10^{6} / \mathrm{ml}$ & $10 \times 10^{6} / \mathrm{ml}$ & Top third & Middle third \\
\hline$\%$ Y count $( \pm 2$ S.E. $)$ & $50 \cdot 1 \pm 3 \cdot 2$ & $50 \cdot 8 \pm 3 \cdot 2$ & $50 \cdot 0 \pm 3 \cdot 2$ & $55 \cdot 7 \pm 3 \cdot 1$ & $67 \cdot 2 \pm 4 \cdot 2$ \\
\hline
\end{tabular}

The increase in motility of spermatozoa after passage through BSA was observed by Ericsson et al. (1973) and Ross et al. (1975) but not by Evans et al. (1975), who did, however, use semen several 
hours after emission and a different method to recover the isolation layers. It is quite clear that an increase was obtained in the present experiments.

As regards the enrichment in Y-bearing spermatozoa, Ericsson et al. (1973) found an increase of up to $85 \%$, whereas Ross et al. (1975) and Evans et al. (1975) observed no increase at all. The enrichment obtained in our experiments, although only modest, is almost certainly real. Because the final score was only a little above $50 \%$, it could be argued that the difference found was due to a bias on the part of the observers, to the fact that the $Y$ count is easier to perform after separation, or because there are fewer abnormal spermatozoa after separation. The last two possibilities may be dismissed, however, as the final score obtained was significantly higher than $50 \%$. The blind precautions taken and the simulation experiments which have been described in detail provide convincing arguments against the first possibility. That the gain obtained was small is not without interest. The fact that neither Ross et al. (1975) nor Evans et al. (1975) obtained any increase may well be due to slight variations in the experimental conditions, such as migration time and the level in the column from which the sample was drawn. In addition, it is possible that such parameters depend upon the subject and it should be noted that the subjects studied by Ericsson et al. (1973) were all carefully selected.

The differential migration of the $\mathrm{X}$ - and $\mathrm{Y}$-bearing spermatozoa remains to be explained. Ericsson et al. (1973) suggested that there may be a difference in motility. Goodall \& Roberts (1976) have now demonstrated that $Y$-bearing spermatozoa are more motile than $\mathrm{X}$, perhaps because of the difference in DNA content. The results obtained in our study for the spermatozoa having two F-bodies provide further information about differential migration. The increase in Y-bearing spermatozoa was accompanied by a significant increase in the percentage of $2 \mathrm{~F}$-bodies (counted with respect to the total number of spermatozoa), the mean values changing from approximately $2 \%$ to $4 \%$. However, when the number of $2 F$-bearing spermatozoa are counted as a percentage of the $Y$-bearing spermatozoa, there was only a slight increase in this value throughout the separation experiments, indicating selection in favour of $2 \mathrm{~F}$ rather than $\mathrm{F}$. There was an observer effect for the $2 \mathrm{~F}$ count in most of the experiments but in the last experiment, when the observers had acquired considerable experience, this proportion increased significantly after separation $(5.9 \%$ versus $3.5 \% ; P<0.05)$. This observation, if confirmed, suggests that even if spermatozoa with $2 \mathrm{~F}$ bodies are not mainly $\mathrm{YY}$-bearing, as is suggested by Beatty (1974a, b), Roberts \& Goodall (1976) and Sumner \& Robinson (1976), at least they differ physiologically from the F-bearing spermatozoa.

\section{References}

Barlow, P. \& Vosa, C.G. (1970) The Y chromosome in human spermatozoa. Nature, Lond. 226, 961962.

BeAtTy, R.A. (1974a) Sperm diversity within the species. In The Functional Anatomy of the Spermatozoon, pp. 319-327. Ed. B. Afzelius. Pergamon Press, Oxford.

BEATTY, R.A. (1974b) Genetic aspects of spermatozoa. In Physiology and Genetics of Reproduction, Part. A, pp. 183-196. Eds E. M. Coutinho \& F. Fuchs. Plenum Publishing Corporation, New York.

Ericsson, R.J., Langevin, C.N. \& Nishino, M. (1973) Isolation of fractions rich in human $Y$ sperm. Nature, Lond. 246, 421-424.

Evans, J.M., Douglas, T.A. \& Renton, J.P. (1975) An attempt to separate fractions rich in human $Y$ sperm. Nature, Lond. 253, 352-354.
Goodall, H. \& Roberts, A.M. (1976) Differences in motility of human $X$ - and Y-bearing spermatozoa. J. Reprod. Fert. 48, 433-436.

Roberts, A.M. \& Goodall, H. (1976) Y chromosome visibility in quinacrine-stained human spermatozoa. Nature, Lond. 262, 493-494.

Ross, A., Robinson, J.A. \& Evans H.J. (1975) Failure to confirm separation of $\mathrm{X}$ - and $\mathrm{Y}$-bearing human sperm using BSA gradients. Nature, Lond. 253, 354 355.

Sumner, A.T. \& Robinson, J.A. (1976) A difference in dry mass between the heads of $\mathrm{X}$ - and $\mathrm{Y}$-bearing human spermatozoa. J. Reprod. Fert. 48, 9-15.

SumNer, A.T., Robinson, J.A. \& EvaNS, H.J. (1971) Distinguishing between $X, Y$ and $Y Y$-bearing human spermatozoa by fluorescence and DNA content. Nature, New Biol. 229, 231-233. 